\title{
Absence of External Causes of Lag in Saccharomyces cerevisiae
}

\author{
By N. B. PAMMENT AND R. J. HALL \\ Department of Biological Process Engineering, School of Chemical Engineering, University of \\ New South Wales, P.O. Box 1, Kensington, New South Wales 2033, Australia
}

(Received 23 November 1977)

\begin{abstract}
In contrast to observations by previous workers of an inverse relationship between inoculum size and the length of the lag phase, the lag time of Saccharomyces cerevisiae was found to be independent of inoculum size whether the inocula were derived from an exponential phase, diauxic lag phase or stationary phase parent culture. It is concluded that the lag phase of this organism is dependent on the internal physiological state of the parent culture cells, rather than the need to accumulate diffusible substances in the extracellular medium.
\end{abstract}

\section{INTRODUCTION}

The initial lag phase in batch culture growth has been ascribed to a variety of causes and is still imperfectly understood. Chesney (1916) first suggested the classification of proposed causes of lag as either internal or external. Internal causes require intracellular adjustments only during the lag phase, such as the need for RNA synthesis or enzyme induction. External causes require changes in the extracellular medium, for example, the accumulation of $\mathrm{CO}_{2}$ or some other diffusible metabolite. In this paper we examine the role of external causes in the lag phase of Saccharomyces cerevisiae.

The principal evidence for the existence of external causes of lag comes from the many reports of an inverse relationship between lag time and inoculum size (e.g. Penfold, 1914; Lodge \& Hinshelwood, 1943; Mian, Prokop \& Fencl, 1971; Kawashima, 1973). This suggests a co-operative effect of the cells in ending lag, either through secretion of a lagreducing substance into the culture fluid or, conceivably, removal of a lag-promoting substance from it. Cultures of higher initial population density show shorter lag phases because more cells are contributing to the extracellular pool of active substance and the critical concentration needed for growth is attained more rapidly. The active substance has been thought to be carbon dioxide (Walker, 1932; Repaske, Repaske \& Mayer, 1974), a range of low molecular weight metabolic intermediates (Lamanna, Mallette \& Zimmerman, 1973) and a specific division-initiating compound, 'schizokinen', which acts as a specific chelator of ferric ions (Lankford et al., 1966; Byers, Powell \& Lankford, 1967; Mullis, Pollack \& Neilands, 1971).

\section{METHODS}

Organism. Saccharomyces cerevisiae 248 unsw 703100 (formerly Saccharomyces cerevisiae Y31), a baker's yeast strain, was used. In this strain the buds separate from the parent cells immediately upon maturation, allowing accurate estimates of cell numbers by plate counts or using a Coulter counter.

Medium. The growth medium contained (per litre distilled water): $5.0 \mathrm{~g}$ glucose, $1.7 \mathrm{~g} \mathrm{KH}_{2} \mathrm{PO}_{4}, 0.056 \mathrm{~g}$ $\mathrm{Na}_{2} \mathrm{HPO}_{4} .12 \mathrm{H}_{2} \mathrm{O}, 3.88 \mathrm{~g}\left(\mathrm{NH}_{4}\right)_{2} \mathrm{SO}_{4}, 0.0755 \mathrm{~g} \mathrm{MgSO}_{4} .7 \mathrm{H}_{2} \mathrm{O}, 9 \cdot 0 \mathrm{~g} \mathrm{NaCl}$ (as an electrolyte), $1 \mathrm{mg}$ inositol, $0.1 \mathrm{mg}$-aminobenzoic acid, $0.2 \mathrm{mg}$ nicotinic acid, $0.2 \mathrm{mg}$ calcium pantothenate, $0.2 \mathrm{mg}$ pyridoxine hydrochloride, $0 \cdot 1 \mathrm{mg}$ riboflavin- $5^{\prime}$-phosphate, $0.2 \mathrm{mg}$ thiamin hydrochloride, $1 \mu \mathrm{g}$ biotin and $0.625 \mathrm{ml}$ mineral salts solution. The mineral salts solution contained (per litre distilled water): $1 \cdot 12 \mathrm{~g} \mathrm{CaO}, 0 \cdot 40 \mathrm{~g} \mathrm{ZnO}, 3 \cdot 24 \mathrm{~g}$ $\mathrm{FeCl}_{3}$ (anhydrous), 1.0 $\mathrm{g} \mathrm{MnCl}_{2} .4 \mathrm{H}_{2} \mathrm{O}, 0.17 \mathrm{~g} \mathrm{CuCl}_{2} .2 \mathrm{H}_{2} \mathrm{O}, 0.24 \mathrm{~g} \mathrm{CoCl} .6 \mathrm{H}_{2} \mathrm{O}, 0.06 \mathrm{~g} \mathrm{H}_{3} \mathrm{BO}_{3}, 13.0 \mathrm{ml}$ $\mathrm{HCl}(10 \mathrm{M})$. The medium was adjusted to $\mathrm{pH} 5 \cdot 2$ and filter-sterilized. 
Culture methods. Four identically equipped fermenters were used - a parent culture fermenter and three subculture fermenters. Quickfit cylindrical glass vessels (working volume $900 \mathrm{ml}$ ) with matching glass flanges were used as the culture vessels. Each fermenter was maintained at $30 \cdot 00 \pm 0.01{ }^{\circ} \mathrm{C}$ by an infrared lamp controlled by a contact thermometer housed in a well. Cooling water was passed continuously through a water jacket. The fermenters were stirred by Toyo magnetic stirrers with $2.5 \mathrm{~cm}$ Teflon stirring bars adjusted by stroboscope to rotate at $1000 \mathrm{rev} . \mathrm{min}^{-1}$. In one experiment (Fig. 5) the parent culture fermenter was agitated by a Vibro-Mischer aerator-stirrer and its oxygen tension was measured with an autoclavable oxygen electrode (Elsworth, 1972). Compressed air was passed to each vessel through a sintered glass sparger. Unless otherwise stated, the air flow was maintained at a constant rate of $100 \mathrm{ml} \mathrm{min}$. $^{-1}$ Loss of material through evaporation was normally negligible: when long culture times were involved, a chilled-water condenser was placed in the effluent gas line. In three experiments (Figs 2 and 5) the $\mathrm{pH}$ of the parent culture was automatically controlled at $5 \cdot 2$ using an autoclavable remote liquid junction calomel electrode (Radiometer K418), a non-autoclavable glass electrode (Radiometer G202C), a pH meter (Radiometer PHM51), an on-off controller (Radiometer Titrator TTT110), a relay and a rotary peristaltic pump which added $0.5 \mathrm{M}-\mathrm{NaOH}$ from a storage vessel.

Preparation of inocula. Stock cultures were maintained on malt agar slopes at $4{ }^{\circ} \mathrm{C}$ and transferred at 8 week intervals. Before each experiment the organism was serially subcultured for at least 100 generations in $250 \mathrm{ml}$ conical flasks containing $50 \mathrm{ml}$ growth medium. The flasks were incubated at $30^{\circ} \mathrm{C}$ on a reciprocating shaker (130 strokes $\left.\mathrm{min}^{-1}\right)$. Transfers were made as often as necessary to keep the cells permanently in the first exponential phase. Approximately $30 \mathrm{ml}$ of an early exponential phase shake-flask culture was then

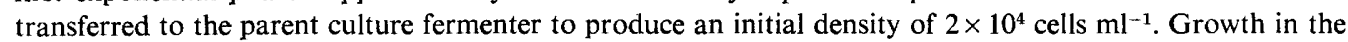
parent culture fermenter was followed using a Coulter counter and was found to be reproducible (Pamment, 1975). At defined points in the growth of the parent culture, inoculations were made into one or more of the three subculture fermenters where the lag phase estimations were made. Transfers were made with warmed fast-delivery pipettes or syringes.

Shock-free inoculation apparatus. In one experiment a vessel-within-a-vessel (Fig. 1) was used to test the effect of eliminating possible inoculation shocks. A self-contained glass 'inoculum tube' (working volume $80 \mathrm{ml}$ ) was inserted through the lid of one of the fermenters. A parent culture grown in the inoculum tube could be subcultured directly into the surrounding medium in the fermenter by breaking the bottom of the tube. The inoculum tube was sealed by an autoclavable rubber stopper through which passed several probes, namely, (i) a gas sparger and gas outlet, similar to those on the fermenters, attached to cotton-wool filters; (ii) a glass capillary tube sealed with a silicone rubber cap through which a syringe was inserted for inoculation and sampling; and (iii) a pointed glass rod which could be forced down through the stopper to break the bottom of the tube. The inoculum tube was filled with sterile medium through a T-junction in the gas inlet line. The exterior vessel served as a constant temperature bath for the inoculum tube; under operating conditions there was no detectable temperature difference between the interior and exterior vessels. The inoculum tube was aerated at $10 \mathrm{ml} \mathrm{min}{ }^{-1}$ and was stirred by a magnetic stirrer bar turned by the magnetic stirrer of the fermenter. Before inoculation, the exterior vessel was stirred by a vigorous stream of sterile air; after breaking the inoculum tube, the stirring bar was released and used to stir the fermenter. The inoculum tube was then raised above the liquid level in the fermenter.

Measurement of growth. Total cell count was measured with a Coulter counter model A (medical) using a $70 \mu \mathrm{m}$ aperture tube. The electrical conductivity of the growth medium was sufficiently high to allow cells in the growth medium to be counted directly. Dilutions, when necessary, were also made with growth medium. Mean cell volume was estimated from frequency distribution histograms of cell volume (Pamment, 1975). At population densities too low for Coulter counter estimations, spread plate counts were made in quadruplicate on malt agar. Dry weight was estimated from triplicate $5 \mathrm{ml}$ samples of the culture, which were centrifuged, washed and recentrifuged twice with distilled water, and dried at $103{ }^{\circ} \mathrm{C}$ for $72 \mathrm{~h}$ before weighing. Percentage viability was estimated by direct microscopic count using methylene blue (Pierce, 1970). Viabilities of all cultures in these experiments were above $96 \%$.

Estimation of glucose. The glucose content of the medium was analysed colorimetrically (Dubois et al., 1956).

Estimation of length of the lag phase. The usuai absence of a well-defined transition point between the lag and exponential phases precludes reliable estimation of the absolute length of the lag phase. Hinshelwood's 'lag time' (Lodge \& Hinshelwood, 1943) provides a more accurate measure of lag and is used here. Lag time, $L$, is defined as the difference between the observed time, $t$, taken for an inoculum of size $N_{0}$ to reach a certain density $N$ in the exponential phase of growth, and the 'ideal time' that would have been taken had the culture commenced growing immediately at the maximum specific growth rate, $\mu_{\max }$ :

$$
L=t-\frac{2 \cdot 303}{\mu_{\max }} \log _{10} \frac{N}{N_{0}}
$$




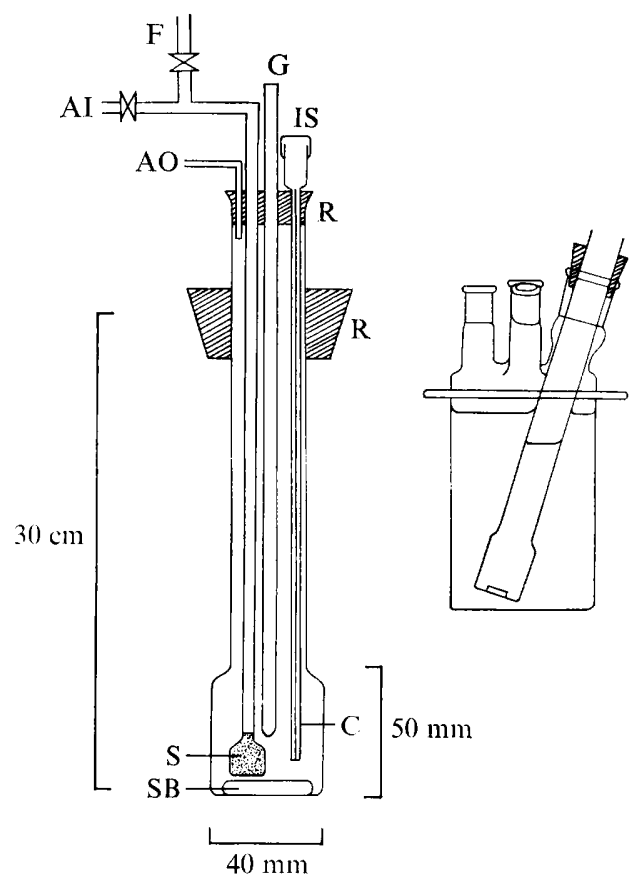

Fig. 1. Shock-free inoculation apparatus (not to scale). Left: inoculum tube showing probes. Right: arrangement of inoculum tube in fermenter. AI, Air in from filter; AO, air out to filter; C, capillary tube; F, filling line from medium reservoir; G, glass rod; IS, inoculation and sampling point; $R$, rubber stopper; $\mathrm{S}$, sparger; $\mathrm{SB}$, stirrer bar.

Lag times were calculated from the formula using values of $\mu_{\max }, t$ and $N$ determined from a least-squares fit of the exponential phase portions of the growth curves. $L$ can also be determined graphically using the extrapolation method shown in Figs 2, 3, 4 and 6.

\section{RESULTS AND DISCUSSION}

The size of the inoculum had no effect on the lag times of three subcultures derived from a diauxic lag phase parent culture, age $20 \mathrm{~h}$ (Fig. $2 a$ ). (The term 'diauxic lag phase' denotes the period, usually 2 to $4 \mathrm{~h}$, separating the phase of fermentative growth on glucose from the later period of respiratory growth on ethanol.) The volume of parent culture fluid carried over with the cells was held constant by adding the appropriate quantities of filtersterilized parent culture fluid to the two smaller inocula. [This precaution was omitted in subsequent experiments since small variations in the amount of parent culture fluid transferred were found to have no detectable influence on lag time (Pamment, 1975).]

Using inocula from a stationary phase culture, age $49 \mathrm{~h}$, the lag times with the two larger inocula were identical but with the smallest inoculum the lag was apparently shorter (Fig. $2 b$ ). This shorter lag time is difficult to understand but may reflect the lower accuracy of plate count determinations compared with those obtained using a Coulter counter. Whatever its significance, the result is clearly opposite to that predicted if the cause of lag were external.

These results contrast with the general observation by previous workers of an inverse relationship between inoculum size and the lag phase. It is possible that the operation of external causes of lag in subcultures from the diauxic lag phase and the stationary phase 


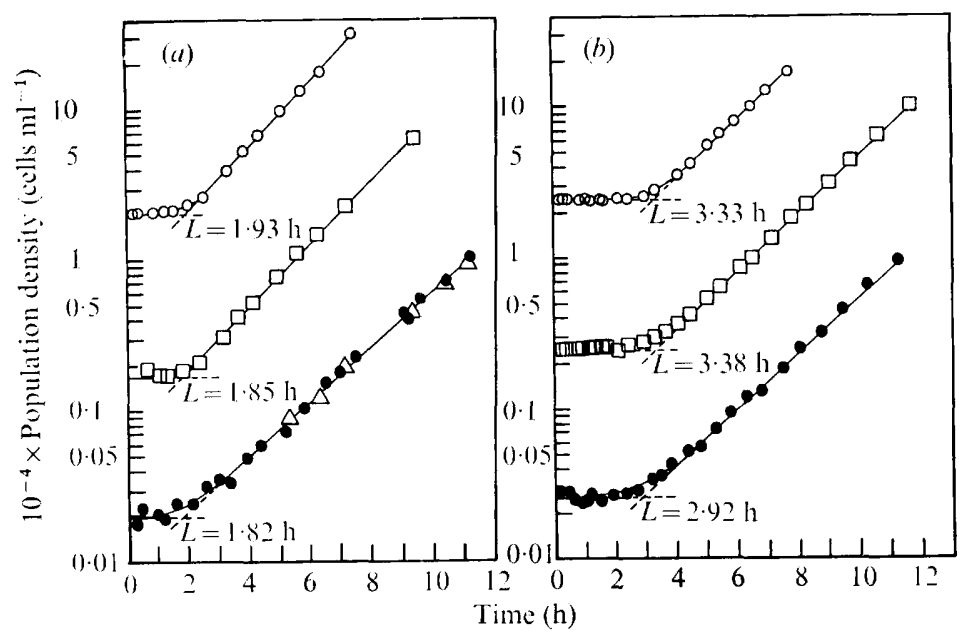

Fig. 2. Effect of inoculum size on the lag time of $S$. cerevisiae subcultures originating from (a) diauxic lag phase parent culture and $(b)$ stationary phase parent culture. In each experiment the parent culture (growth not shown) was controlled at $\mathrm{pH} 5 \cdot 2 ; \mathrm{pH}$ in the three subculture fermenters was uncontrolled. Cell numbers were estimated with the Coulter counter $(O, \square, \triangle)$ and by spread plate counts $(\mathbf{O})$.

was obscured by the predominance of relatively severe internal lag producing factors [e.g. the data of Beck \& von Meyenburg (1968) indicate that derepression of glycolytic enzymes would be required]. For this reason, subsequent experiments were performed with early-exponential phase inocula, which should fundergo relatively few internal readjustments on subculture, allowing external causes of lag to be examined in comparative isolation.

Inocula taken from exponentially growing cultures are often claimed to grow without lag, provided the temperature and medium composition are not changed on subculture (Lamanna et al., 1973; Brock, 1974; Wilson \& Miles, 1975). Finite lag times have however been reported, ranging from $0.5 \mathrm{~h}$ (Taylor, 1954; Duncan \& Nickerson, 1963) to several hours (Stern \& Frazier, 1941 ; Lodge \& Hinshelwood, 1943; Walker, 1963). Walker (1963) found that the lag time of exponential phase subcultures of Bacillus megaterium was inversely proportional to inoculum size.

Two inocula (varying 100-fold in size) taken from an early-exponential phase culture of $S$. cerevisiae both showed a drop in growth rate immediately after transfer, giving rise to lag times of 0.49 and $0.70 \mathrm{~h}$ (Fig. 3). Short but definite lag phases of this kind were observed in all experiments with exponential phase inocula of this organism (Pamment, 1975). The apparent difference in lag time is slight compared with the large difference in inoculum size, making it improbable that an external cause alone was the lag-producing factor.

A necessary (but not sufficient) test for the existence of external causes of lag is whether lag is reduced when the growth medium is supplemented with the spent or partially spent medium obtained by filtering previous cultures. Since culture filtrates should contain the hypothetical lag-reducing substance, they should eliminate any lag due to an external cause alone and reduce the lag time, where an external cause is contributory. Small supplements of sterile filtrates eliminated the lag of exponential phase inocula of Aerobacter aerogenes (Lodge \& Hinshelwood, 1943) and B. megaterium (Walker, 1963). Figure 4 shows the effect of filtrates taken from the early-exponential phase ('early' filtrate) and the late-exponential phase ('late' filtrate) from previous cultures, on the lag time of exponential phase inocula of $S$. cerevisiae. No significant shortening of the lag occurred in the 'late' filtrate, but a slight reduction $(0 \cdot 14 \mathrm{~h})$ was observed for the culture in $100 \%$ 'early' filtrate. (The maximum 


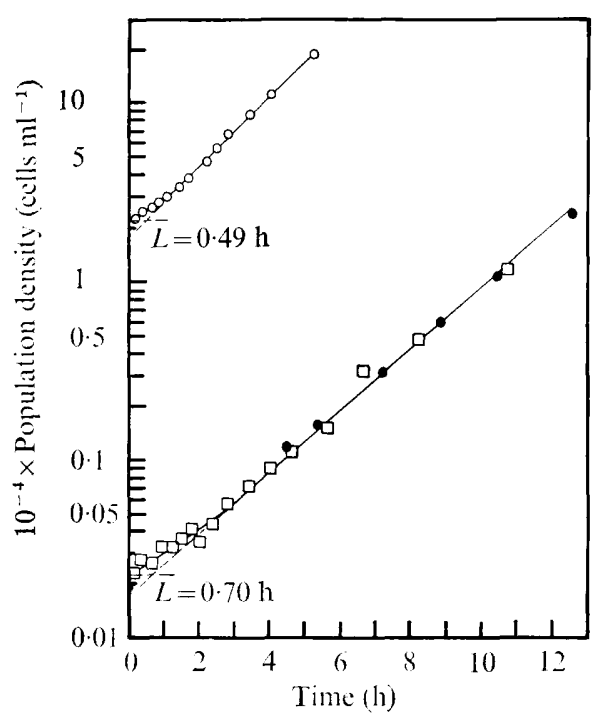

Fig. 3

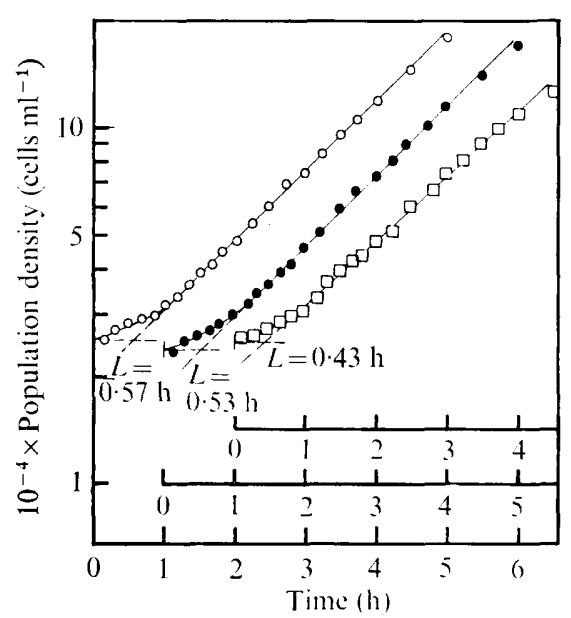

Fig. 4

Fig. 3. Effect of inoculum size on the lag time of $S$. cerevisiae subcultures derived from an earlyexponential phase parent culture (age $8 \mathrm{~h}$, population density $8 \times 10^{5}$ cells $\mathrm{ml}^{-1}$ ). Cell numbers were estimated by Coulter counter $(O, 0)$ and spread plate counts $(\square)$.

Fig. 4. Effect of filter-sterilized medium from previous cultures on the lag time of S. cerevisiae. Subcultures were inoculated simultaneously from an early-exponential phase parent culture (population density $9 \times 10^{5}$ cells $\mathrm{ml}^{-1}$ ) into: $\bigcirc$, fresh growth medium (control); $\bigcirc$, growth medium supplemented with $10 \%(\mathrm{v} / \mathrm{v})$ 'late' filtrate from a late exponential phase culture (population density $10^{7}$ cells $\left.\mathrm{ml}^{-1}\right) ; \square, 100 \%$ 'early' filtrate from an early-exponential phase parent culture (population density $9 \times 10^{5}$ cells $\mathrm{ml}^{-1}$ ). Time scales are staggered for presentation purposes.

specific growth rate of this culture was also slightly reduced for unknown reasons; the reduction did not occur in subsequent comparable experiments.) Similar small reductions $(0.12,0.109$ and $0.12 \mathrm{~h})$ in lag time in 'early' filtrate were observed in three further experiments (Pamment, 1975), confirming that the small difference is real.

The failure of the 'early' or 'late' filtrates to eliminate the lag phase rules out an external cause as the only cause of lag for exponential phase inocula. (The possibility that the extracellular substance was unstable or gaseous is discounted in a subsequent experiment.) The slight reduction in lag time with 'early' filtrate may indicate the presence of an external cause of lag in a minor contributory role. However, since the lag time could also have been reduced simply because of a change in whatever condition is responsible for the lag, no positive evidence of an external cause has been produced, and we conclude that the cause of lag is purely internal. Two further observations support this argument. Firstly, 'late' filtrate failed to reduce the lag time. This may be compared with the significant reduction in lag time with 'late' filtrates for organisms which do require the secretion of a lag-reducing substance (Lodge \& Hinshelwood, 1943; Lankford et al., 1966; Halmann, Benedict \& Mager, 1967). Secondly, the lag time of exponential phase subcultures increased severalfold as the age of the parent culture increased through the exponential phase (Fig. 5 ; also Taylor, 1954). As the initial cell densities of the subcultures were all the same, a lag caused by external causes would be expected to be essentially constant.

Our inability to detect external causes of lag in exponential, diauxic lag and stationary phase subcultures leads to the general conclusion that the length of lag depends only on the internal physiological condition of the inoculum cells and this was verified experimentally (Fig. 5). The parent culture showed the typical diauxic pattern observed by previous workers 


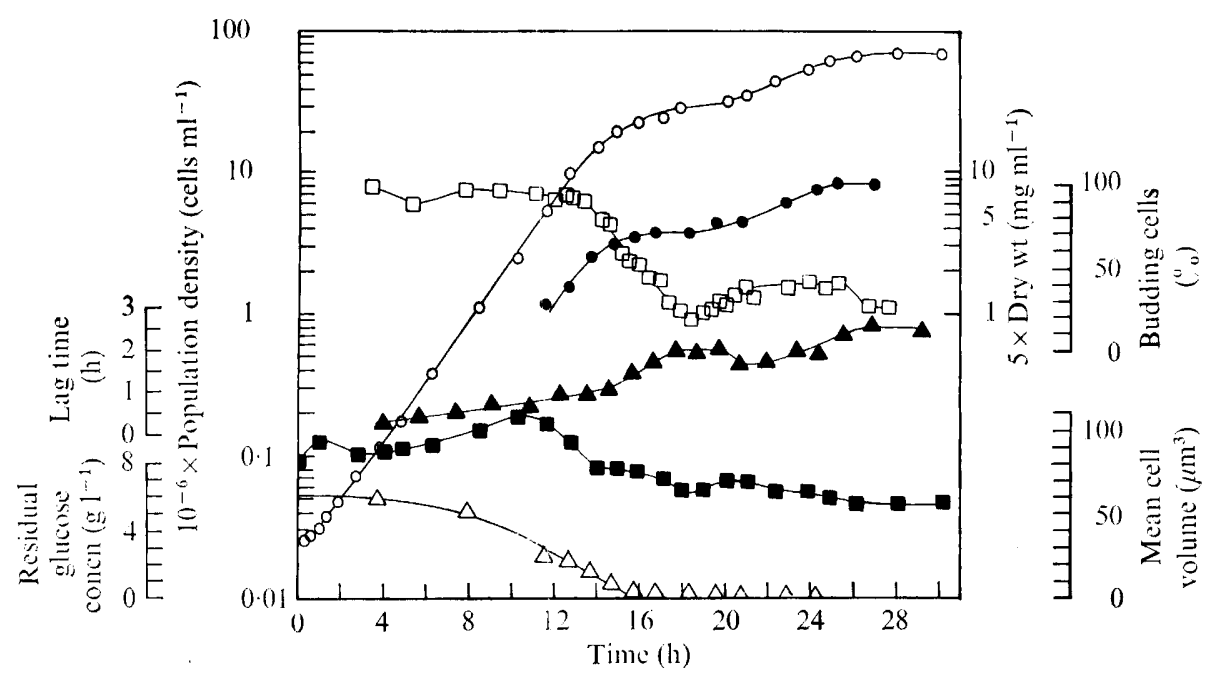

Fig. 5. Dependence of subculture lag time on the age and growth phase of the parent culture. The parent culture was grown in a pH-controlled fermenter and estimations were made of cell numbers $(\bigcirc)$, dry weight $(0)$, percentage of budding cells $(\square)$, mean cell volume $(\square)$ and residual glucose concentration $(\triangle)$. Throughout the growth of the parent culture, shake-flask subcultures of initial density $2.5 \times 10^{4}$ cells $\mathrm{ml}^{-1}$ were made into fresh glucose medium and their lag times $(\boldsymbol{\Delta})$ were determined from Coulter counter estimates of cell numbers.

(Beck \& von Meyenburg, 1968; Bijkerk \& Hall, 1977) for aerobic culture of S. cerevisiae, namely, an initial, rapid, glycolytic growth phase, during which glucose was assimilated and ethanol excreted, followed by a diauxic lag phase and then a slower respiratory growth phase on the accumulated ethanol. During the first growth phase, the increase in lag time was slight since the metabolism of the parent culture remained predominately glycolytic throughout this period. The slow increase in lag time reflected the incompletely balanced nature of exponential growth in batch culture, as indicated by the variations in mean cell volume and percentage of budding cells. With the exhaustion of glucose, a rapid increase in lag time was observed, attributable both to the rapid run-down in metabolic activity of the parent culture after the energy source was consumed, and the gradual transition of enzyme activity from a glycolytic to a respiratory pattern (Beck \& von Meyenburg, 1968). The fall in lag time observed towards the beginning of respiratory growth was probably due to the resurgence of metabolic activity after the diauxic lag period. The phase of respiratory growth was marked by a rapid increase in the activities of the enzymes of the tricarboxylic acid cycle and oxidative phosphorylation, and a decrease in glycolytic enzyme activities (Beck \& von Meyenburg, 1968). Accordingly, the lag time of inocula taken from such cultures increased. While no increase in lag time was observed after the cessation of growth of the parent culture in this experiment, a gradual increase, attributable to a slow deterioration of the cell contents, was observed in a similar experiment (Pamment, 1975) in which lag time was followed further into the stationary phase. While a multiplicity of internal lag producing factors probably combine to determine the lag phase, most of the trends in Fig. 5 can be predicted by a mathematical model (Pamment, Hall \& Barford, 1978) which assumes that the major cause of lag for subcultures in glucose medium is the need to restore the activities of the glycolytic enzymes to their optimum levels for fermentative growth. Figure 5 may be contrasted with the lag-age curve of Lodge \& Hinshelwood (1943) for an organism whose primary cause of lag is external.

The experiment of Fig. 4 raises the side-issue of the cause of lag for cultures taken from the exponential phase and inoculated into 'early' filtrates. Cultures grown in 'early' filtrates 


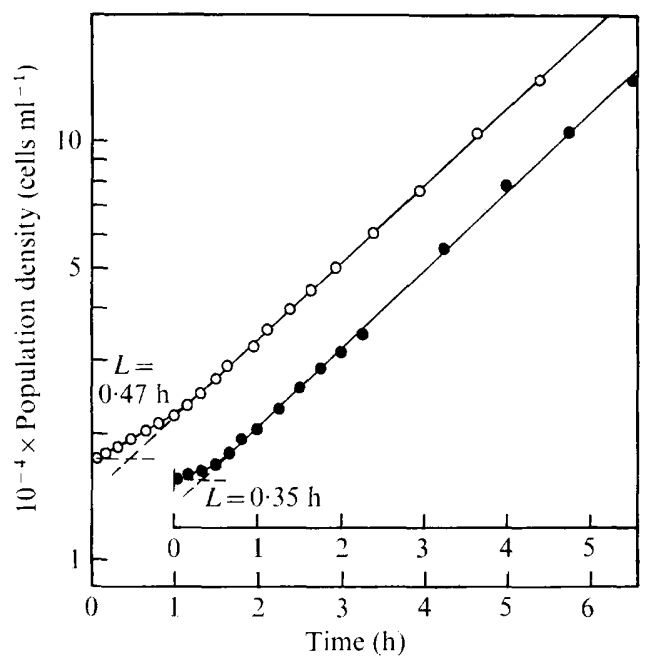

Fig. 6. Effect of eliminating inoculation shocks on the lag time of exponential phase subcultures of $S$. cerevisiae. Separate early-exponential phase parent cultures were grown in the inoculum tubes of two shock-free inoculation devices (Fig. 1) and inoculated simultaneously into exterior vessels containing: $\bigcirc$, fresh medium; $\bigcirc$, 'early' filtrate.

(see also Pamment, 1975) showed lag times only slightly shorter than those in fresh medium, the lag phase not being eliminated completely. This result is surprising because the inocula were transferred from parent cultures of population density $9 \times 10^{5} \mathrm{cells} \mathrm{ml}^{-1}$ to filtrates prepared from cultures of the same population density. There should have been no detectable difference in the compositions of the parent culture and subculture media, and the cells should have shown no lag. It is conceivable that active substances are lost from the 'early' filtrate before use, either through decomposition of unstable materials, or desorption of dissolved $\mathrm{CO}_{2}$, a proven lag-controlling factor in some previous studies (Walker, 1932; Repaske et al., 1974). These explanations were dismissed for the present system, since subcultures in fresh 'early' filtrate, prepared $30 \mathrm{~min}$ before use, or supplemented with $\mathrm{CO}_{2}$ $[10 \%(\mathrm{v} / \mathrm{v})$ in air] failed to show any additional reduction in lag time (Pamment, 1975).

An alternative explanation of the lag in 'early' filtrates may be the shocks (temperature, mechanical or otherwise) encountered when the cells are transferred from vessel to vessel. To test this hypothesis, an inoculation apparatus was constructed (see Methods) which allowed practically instantaneous inoculation with no possibility of temperature shock or physical damage to the cells. Figure 6 shows the growth of cultures in fresh medium and 'early' filtrate inoculated in this manner. The lag time in 'early' filtrate was, as usual, less than that of the control. However, in neither case was the lag phase eliminated and the observed lag times were not significantly shorter than those found in previous experiments. The experiment fails to support the hypothesis that the lag in 'early' filtrates is due to transfer shocks and its true cause remains unknown. 


\section{REFERENCES}

Beck, C. \& von MeyenburG, H. K. (1968). Enzyme pattern and aerobic growth of Saccharomyces cerevisiae under various degrees of glucose limitation. Journal of Bacteriology 96, 479-486.

Bijkerk, A. H. E. \& Hall, R. J. (1977). A mechanistic model of the aerobic growth of Saccharomyces cerevisiae. Biotechnology and Bioengineering 19, 267-296.

Brock, T. D. (1974). Biology of Microorganisms, 2nd edn, p. 238. Englewood Cliffs, New Jersey: Prentice-Hall.

Byers, B. R., Powell, M. V. \& Lankford, C. E. (1967). Iron-chelating hydroxamic acid (schizokinen) active in initiation of cell division in Bacillus megaterium. Journal of Bacteriology 93, 286-294.

Chesney, A. M. (1916). The latent period in the growth of bacteria. Journal of Experimental Medicine 24, 387-409.

Dubois, M., Gilles, K. A., Hamilton, J. K., Rebers, P. A. \& SMith, F. (1956). Colorimetric method for the determination of sugars and related substances. Analytical Chemistry 28, 350-356.

Duncan, D. W. \& Nickerson, J. T. (1963). Effect of environmental and physical conditions on the phase of adjustment of Pseudomonas fragi. Applied Microbiology 11, 179-183.

ELSWORTH, R. (1972). The value and use of dissolved oxygen measurements in deep culture. Chemical Engineer 258, 63-71.

Halmann, M., Benedict, M. \& Mager, J. (1967). Nutritional requirements of Pasteurella tularensis for growth from small inocula. Journal of General Microbiology 49, 451-460.

KaWASHIMA, E. (1973). Mathematical model taking account of the lag phase in microbial growth. Journal of Fermentation Technology 51, 41-46.

Lamanna, M., Mallette, M. F. \& Zimmerman, L. N. (1973). Basic Bacteriology. Its Biological and Chemical Background, 4th edn, pp. 357-363. Baltimore: Williams \& Wilkins.

LANkford, C. E., WAlker, J. R., Reeves, J. B., NABbUt, N. H., BYERS, B. R. \& JONES, R. J. (1966). Inoculum-dependent division lag of Bacillus cultures and its relation to an endogenous factor(s) (schizokinen). Journal of Bacteriology 91, 1070-1079.
LOdGE, R. M. \& HinsHelWOOD, C. N. (1943). Physicochemical aspects of bacterial growth. IX. The lag phase of Bact. lactis aerogenes. Journal of the Chemical Society, 213-219.

Mian, F. A., Prokop, A. \& Fencl, Z. (1971). Growth and physiology of a yeast cultivated in batch and continuous culture systems. Folia microbiologica 16, 249-259.

Mullis, K. B., Pollack, J. R. \& Neilands, J. B. (1971). Structure of schizokinen, an iron-transport compound from Bacillus megaterium. Biochemistry 10, 4894-4898.

Pamment, N. B. (1975). The lag phase in microbial growth. Ph.D. thesis, University of New South Wales, Australia.

Pamment, N. B., Hall, R. J. \& Barford, J. P. (1978). Mathematical modelling of lag phases in microbial growth. Biotechnology and Bioengineering (in the Press).

Penfold, W. J. (1914). On the nature of the bacterial lag. Journal of Hygiene 14, 215-241.

Pierce, J. S. (1970). Measurement of yeast viability. Journal of the Institute of Brewing 76, 442-443.

Repaske, R., Repaske, A. C. \& MAYER, R. D. (1974). Carbon dioxide control of lag period and growth of Streptococcus sanguis. Journal of Bacteriology 117, 652-659.

Stern, R. M. \& Frazier, W. C. (1941). Physiological characteristics of lactic acid bacteria near the maximum growth temperature. Journal of Bacteriology 42, 479-499.

TAYLOR, H. E. (1954). A study of the lag phase in the growth of yeast cultures. Revue canadienne de biologie 13, 144-169.

WALKER, H. H. (1932). Carbon dioxide as a factor affecting lag in bacterial growth. Science 76, $602-604$.

WALKER, J. R. (1963). Dependence of cell division of Bacillus megaterium on an endogenous compound. Ph.D. thesis, University of Texas, U.S.A.

Wilson, G.S. \& Miles, A. (1975). Topley and Wilson's Principles of Bacteriology, Virology and Immunity, 6th edn, vol. I, p. 122. London: Arnold. 